\title{
A Safe and Reliable Method for Installing Wireless LAN into a Hospital
}

\author{
Eisuke Hanada ${ }^{1} \cdot$ Takato Kudou ${ }^{2}$
}

\begin{abstract}
A medical environment in which patient information can be accessed anytime / anywhere is called a "ubiquitous environment". To realize such an environment, the installation of wireless LAN is quite effective. Because the maximum radio wave output (antenna power) is set low in Japan, it has been easy to safely introduce wireless LAN into hospitals, to date mainly into large hospitals. However, if the placement of access points is not done properly, problems will occur, such as signals not reaching the desired area. A solution to these types of problems is to do an electromagnetic-field propagation simulation, which should be performed before construction of the hospital. It is also necessary to protect against security problems, such as signal interception or illegal access. We herein show our procedures for the safe introduction of wireless LAN.
\end{abstract}

Key words: Wireless LAN, Electromagnetic Propagation Simulation, Shielding, Security, User Education.

\section{Demand for Wireless LAN in Hospitals}

The introduction of wireless LAN enables not only the input of information at the point information is generated, but the construction of an environment in which "information can be referred to anytime / anywhere". If this environment is realized, it is called a "ubiquitous environment".

As modern clinical medicine has become more complex in large hospitals, it has become difficult for doctors alone to treat patients. The concept of "clinical team" is widespread in large Japanese hospitals. A team consists of doctors and the co-medical staff working with them. Each team member shares information, and plays a specific role in patient care. In large Japanese hospitals, patient information, including electronic patient charts are often stored in the computer (server) hosting the hospital information system (HIS). Therefore, even if team members are not in close proximity, it is possible for them to refer to information using remote terminals. The introduction of wireless LAN into a hospital allows the quick, safe, and reliable sharing of patient information, and the demand for wireless data communication systems is expected to increase in the future. Realizing a "ubiquitous environment" in a hospital is quite effective for improving labor efficiency.

Recently, the number of Japanese hospitals that have installed wireless LAN has increased rapidly. However, if the areas in which the electromagnetic signals $(2.45$ $\mathrm{GHz}$ bands and 5.2 $\mathrm{GHz}$ bands) used by wireless LAN are not positioned correctly, terminals will not be able to connect to the HIS. Also, it is necessary to protect against security problems, such as signal interception or illegal access.

In Japan, no guidelines or installation procedures for wireless LAN use in hospitals have been published. We herein show our design for wireless LAN accessible areas, a design that insures that wireless LAN can be safely and reliably installed in hospitals.

\section{Safety of Wireless LAN Use from the Viewpoint of Electromagnetic Interference}

Because wireless LAN uses electromagnetic waves, some hospitals are concerned about electromagnetic interference (EMI) with medical devices by cellular phones, sometimes from prior experience but often from hearsay, and thus hesitate to introduce wireless LAN.

The guidelines for cellular phone use announced by the Electromagnetic Compatibility Committee (Japan) [1] are well known in Japan; however, they were announced before standard wireless LAN specifications were accepted. The Ministry of Internal Affairs and Communications of Japan (MIC) has researched the possibility of EMI with medical devices almost every year since 1996. The studies by the MIC mainly used cellular phones,

\footnotetext{
Manuscript received May 16, 2011 ; revised October 13, 2011. (ID No. 20110516-01J)

${ }^{1}$ Division of Medical Informatics, Shimane University Hospital, Izumo, Japan.

${ }^{2}$ Department of Electrical and Electronic Engineering, Faculty of Engineering, Oita University, Oita, Japan.

Corresponding Author : Eisuke Hanada (e-mail : e-hanada@med.shimane-u.ac.jp)
} 
wireless LAN devices, RF-ID tags (electronic tags), etc. as sources of electromagnetic waves. MIC has released reports about EMI with implantable medical devices annually. In their latest reports [2], they wrote that, in most cases no EMI with medical devices will occur by the use of wireless LAN. This is because the allowed output for wireless LAN instruments is low by Japanese radio law; less than $200 \mathrm{~mW}$, in general.

To the contrary, Hanada et al. have shown experimental data that some medical devices stopped when irradiated with high-output electromagnetic waves of the frequency used by wireless LAN [3]. These results also showed that safety is high if the electromagnetic wave output is weak.

\section{Requirements for Electromagnetic-field Propagation Simulation}

Not only in hospitals, but in any building, wireless communications are affected by the construction materials used in walls, floors, and windows, etc. For example, it is obvious that metal walls would reflect electromagnetic waves; however, the users of buildings often do not know the composition of the construction materials, especially the many materials that cannot be seen.

In the results of experiments by Hanada et al., a $12 \mathrm{~cm}$ thick pressed concrete wall in which steel rods were embedded at $75 \mathrm{~cm}$ intervals had a shielding capability of only $2 \mathrm{~dB}$ to a $1.5 \mathrm{GHz}$ electromagnetic wave. A partition of compressed paper sandwiched between two 0.5 $\mathrm{mm}$ thick aluminum plates had shielding capability no less than $39 \mathrm{~dB}$ [4]. They also showed that stationary waves can be generated indoors when the indoor metal fixtures (especially cabinets) reflected electromagnetic waves [4].

Therefore, if the position of wireless access points (AP) is decided without taking into careful consideration the influence of the above mentioned materials and fixtures before installation, communication failures may occur. One of the most common communication failures is that electromagnetic signals do not reach the desired area. Another example is the appearance of roaming disturbance, which brings connection concentration into one AP.

When constructing a building, communication equipment, such as cables, are installed both during construction and after construction is completed. Because it is difficult to install cables after the use of a hospital building (especially ward) has begun, the placement of communication equipment should be done during construction. It is important that the position of APs be defined before the actual environment is built. Although the ar- chitect is the logical person to determine the position of APs, as has been done in the past, we recommend that electromagnetic-field propagation simulation be done for all future hospital buildings.

\section{Simulation Techniques and an Example of Application}

The Ray-tracing method [5], [6] and the FDTD method [7] are well known electromagnetic-field propagation simulation procedures. Although both are useful and there is dedicated software, calculation takes a great amount of time. When three-dimensional analysis is conducted using the FDTD method, huge computational complexity is required, and several days are necessary for each room simulation, even when a supercomputer is used.

We did a simulation for almost all the floors of the new Shimane University Hospital building (9 floors) before introducing wireless LAN. We were told that the architect planned six APs per floor in his design.

In our new hospital building, an emergency room, a sterilization section, and a pharmacy will be located on the first floor. An intensive care unit (ICU), high care unit (HCU), and medical engineer (ME) center will be on the second floor, and 10 surgical theaters will be on the third floor. The fourth floor is small, so it will be used for equipment storage, with no patient entry allowed. The fifth floor to the ninth floor will be wards. Our simulation for the new hospital, was done using the Dominant Path Model [8], adapting the Ray-tracing method for the purpose of obtaining a bit rate of 10 or more Mbps at bedside in all of the wards. The Dominant Path Model reduces the computational complexity by eliminating paths considered to be unnecessary because of their direction of reflection.

The conditions for the simulation are as follows. The floor of the target space is built by a slab construction method that uses steel rods and concrete. The door of each patient room is lightweight steel. The door of the pipe space (corridor side) for the toilets in the patient rooms is made of iron. Glass does not have any special electromagnetic shielding capacity. The procedure of the simulation is as follows. First, these pieces of rough component information are input and the simulation to compute the number of required AP is done. We then added more component information. For example, doors of the custodial closets in the corridors. We can thus calculate the number and best positions for the APs.

Figure 1 is an example of our results. On this floor, 12 APs are necessary to cover whole area within which notebook-type PCs will be connected to the hospital LAN. Our results showed that sufficient transmission speed at 


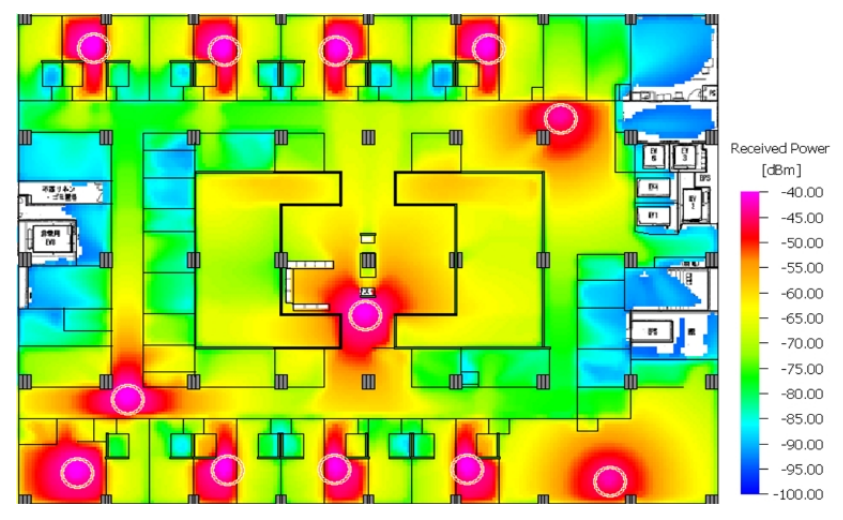

Fig. 1. Simulation result(the fifth floor).

$\mathrm{O}$ : The recommended position of AP

bedside can be obtained by installing 11 to 13 sets of AP per floor.

The LAN wiring of this building was done several months before the installation of the AP. For this reason, some AP had to be installed in a different position from the recommendation position. This resulted from constructing the building without waiting for a simulation. Schedule adjustment is important in order to build a better wireless-communications environment.

A survey of the intensity of electromagnetic waves was done after the building was completed to verify the validity of the results of our simulation and the effect of the difference in AP position. The results showed that the signal was more strongly detected than expected. This means that higher-speed wireless communications are possible at many points. We considered some possible causes. Metal doors had been expected to reach the ceiling, but in fact they were low and made of gypsum board, which allowed the conduction of electric waves, between the top of the door and ceiling. Also, the door in the corridor which we expected would be made of metal was made of glass that lacked shielding capacity.

The influence of the difference in AP position was expected to become smaller, but because the access range was larger than expected, we did not find any effect from the position difference.

\section{Wireless LAN Instllation Method in a Hospital}

When installing wireless LAN into a hospital, the installation procedures must be divided into fundamental planning, design of electromagnetic signal accessible areas, and the arrangement of AP locations.

The person responsible for fundamental planning should determine the following: the purpose of use, the users, the kind of data to be communicated through the wireless LAN, and the functions of the wireless LAN equipment. This determination affects the transmission rate and standards to be adopted.

Also, security functions should be determined at this stage. The security policy should include the installation of filtering to protect against interception, measures to protect against illegal access, and application of authentication technology. If the wireless LAN is exclusively for the hospital staff, adopting cypher technology is recommended. These are included in the guidelines announced by the Ministry of Health, Labour and Welfare of Japan [9]. It is also important to be able to store and check communication logs. However, for users who belong to the staff, it is important to avoid anything that would increase the burden of performing their tasks.

Next, the areas to which electromagnetic signals must reach needs to be defined. In this stage, an electromagnetic propagation simulation should be taken into consideration. Before doing a simulation, the planner should gather as much information as possible about the materials used in the building. Data on the materials, such as permeability, permittivity, position and size of a component, etc., should be carefully gathered, because it greatly affects the results of simulation. The floor-plan of each floor, an elevation rendering of the building, sometimes including CAD data, and the location and materials of fixtures to be installed are necessary to insure the accuracy of the simulation models.

Based on our experience, even if a propagation simulation method is used, it must be kept in mind that simulation results are inexact. First, even if the information about the permeability, permittivity, and conductivity of a component over electromagnetic waves is obtained, the location, thickness, and size of a component may differ from the information on the blueprint and from the actual construction. Next, in a simulation, it is difficult to take into consideration reflection from metal fixtures etc. that are installed after completion and wave absorption by people. In an electromagnetic wave propagation simulation, it is unreal to incorporate medium information that is significantly smaller than the wavelength of the target electromagnetic waves. Therefore, there is an inherent limit in the precision of a simulation result.

The positioning of AP can thus be determined through simulations or by using AP location tools provided by the AP provider. For the final AP location, repeated simulations might be required. In such cases, we feel that for appropriate design and installation to be possible, an electromagnetic wave propagation simulation must be done. In some areas of the hospital it is important that there be no electromagnetic waves, making electromagnetic shielding of such areas absolutely necess- 
ary.

Furthermore, the electric waves of the wireless LAN used outside the hospital may invade and interfere. Therefore, before starting operation, the situation of electric wave invasion needs to be investigated. When interference is found, it may be necessary to add measures, such as putting shielding materials on the walls or windows. If the invading signals are weak, some settings, such as SSID or encryption, will be effective in eliminating interference.

In addition, after introducing wireless LAN, the range of access of the electric waves sometimes changes; for example, with the addition or movement of fixtures or when renovating or dividing a room. There may also be interference to communication by electromagnetic waves emitted in a hospital, depending on the selected standard. Even after the introduction of wireless LAN, the person responsible for the LAN must be flexible and reposition the AP in consideration of changes to the hospital environment.

Even if wireless LAN is designed well, information leaks may occur if a user does not operate the terminal carefully. It is important to educate the users not to leave terminals unattended that are displaying personal information, for example.

Table 1 gives a summary of the procedures. Because they are in the process of development but will be used in the near future, we would appreciate your opinions on how to improve them.

\section{Conclusions}

In this paper, we show simulation results and proposed procedures for the safe introduction of wireless LAN to a hospital. The simulation showed weakness in the construction plan in that the structural architect had insufficient knowledge of electromagnetic environments and that the communication system planner lacked the knowledge necessary to provide the necessary building components.

The clinical staff is encouraged to discuss and comment on the simulation and survey results, even though specialists perform the simulation and survey.

To insure safe and reliable wireless communications in hospitals, we recommend the use of simulation technology at the earliest possible stage of construction. In this way, a stable, ubiquitous environment can be realized without the problems associated with the trial and error type trouble-shooting used too often in the past for systems that have been installed without proper planning.
Table 1. Hospital instllation procedures and the points to be designed.

\begin{tabular}{|l|l|}
\hline 1. Fundamental design \\
\hline & Users of the wireless LAN \\
\hline & $\begin{array}{l}\text { Ex.) Only for the staff or where permitted for } \\
\text { patient use }\end{array}$ \\
Wireless LAN communication \\
\hline $\begin{array}{l}\text { Ex.) For character data only, or movie data } \\
\text { may also available }\end{array}$ \\
\hline \begin{tabular}{l} 
Function \\
\hline
\end{tabular} \\
\hline $\begin{array}{l}\text { Ex.) For data communication, VoIP, positioning, } \\
\text { or a mixture }\end{array}$ \\
\hline $\begin{array}{l}\text { Access security; Ex.) Filtering to avoid inco- } \\
\text { rrect access, or authentification by ID and pass- } \\
\text { word }\end{array}$ \\
\hline $\begin{array}{l}\text { Others; Ex.) Management of the communication } \\
\text { log, Prevention of illegal access using a fire- } \\
\text { wall etc. }\end{array}$ \\
\hline Standards for wireless LAN \\
\hline $\begin{array}{l}\text { Ex.) IEEE 802.11 series, IEEE 802.15 series, } \\
\text { bluetooth, or others }\end{array}$ \\
\hline Designing product specifications \\
\hline \begin{tabular}{l} 
Ex.) AP, router, server, etc. based on the above \\
\hline
\end{tabular}
\end{tabular}

2. Designing areas accessible to the wireless LAN

Rough design

Ex.) Range; inside of a specific room, the whole floor, or the whole building, etc.

Detailed design*

Ex.) Taking furnishings into account and investigation of the wall materials

\section{Arrangement of access point location}

\begin{tabular}{|l|l|}
\hline & Determination of the product \\
\hline & The location design united with the area* \\
\hline & $\begin{array}{l}\text { Ex.) Electromagnetic-propagation simulation, Tools } \\
\text { for plannnng AP location }\end{array}$
\end{tabular}

Wiring and the design of the power supply to APs

4. Verification after installation

Checking for invasive electric waves from the outside and implementation of corrective measures

Ex.) Attachment of sheilding materials to windows and walls

Maintenance of access points

Ex.) Measures to protect against mechanical failure, Adjustment of output

Correction of access point location

Ex.) Extension and alteration, installation of new fixtures

Additional access point installation

Ex.) In the case of a newly divided room

Verification and addition of security

Ex.) Security based on log checks and accident reports

* The time at which the electromagnetic propagation simulation should be done. 
This research is based on the joint research of Shimane University Hospital and KYOCERA Communication System Co., Ltd. It was partially supported by a Japan Society for the Promotion of Science grant-in-aid for scientific research (Basic research (B), No. 20390151). This research also includes the results of the joint research of Shimane University Hospital and Shimizu Corporation.

\section{References}

[1] Electromagnetic Compatibility Committee (Japan), Research Report of the usage of radio-communication equipment such as cellular telephone handsets, Japan, Association of Radio Industries and Business; 1997.

[2] The Ministry of Internal Affairs and Communication (Japan) : Investigation of the Effects of Radio Waves on Medical Equipment etc(http://www.tele.soumu.go. jp/e/sys/ele/medical/index.htm) Reffered on Sep. 2010.

[3] E. Hanada, Y. Hoshino, and T. Kudou, "Safe introduction of in-hospital wireless LAN," Medinfo 2004, pp. 1426-1429, 2004.

[4] E. Hanada, Y. Watanabe, Y. Antoku, Y. Kenjo, H. Nutahara, and Y. Nose, "Hospital construction mate-

Eisuke Hanada

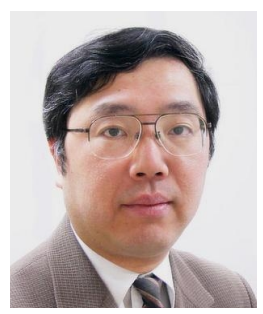

was born in Tokyo, Japan, in 1963. He received his B. Eng. and M. Eng. degrees from Kyushu University, Fukuoka, Japan, in 1985 and 1987, respectively. He received his D. Eng. degree from Saga University, Saga, Japan, in 2001. From 1992, he worked at Nagasaki University Information Science Center for four years managing the campus LAN and information servers. And at the Department of Medical Information Science, Kyushu University Graduate School of Medical Science. Since 2002, he has been working at the Division of Medical Informatics, Shimane University Hospital. His research involves the wired/radio communication environment and information processing systems in hospitals.

Dr. Hanada is a member of the Japanese Society of Medical Informatics, the Information Processing Society of Japan, the Japanese Society for Medical and Biological Engineering, the Healthcare Engineering Association of Japan, and the Acoustical Society of Japan. rials: Poor shielding capacity with respect to signals transmitted by mobile telephones," Biomedical In. Strumentation \& Technology, vol. 35, no. 4, pp. 489496, 1998.

[5] Z. Ji, B-H. Li, H-X. Wang, H-Y. Chen, and T. K. Sarkar, "Efficient ray-tracing methods for propagation prediction for indoor wireless communications," IEEE Antennas and Propagation Magazine, vol. 43, no. 2, pp. 41-49, 2001.

[6] R. Hoppe, P. Wertz, F. M. Landstorfer, and G. Wölfle, "Advanced ray-optical wave propagation modelling for urban and indoor scenarios including wideband properties," European Transactions on Telecommunications, vol. 14, no. 1, pp. 61-69, 2003.

[7] K. S. Yee, "Numerical solution of initial boundary value problems involving maxwell's equations in isotropic media," IEEE Transactions on Antenna Propagation, vol. 14, no. 3, pp. 302-307, 1966.

[8] G. Woelfle, F. M. Landstorfer, "Dominant paths for the field strength prediction," 48th IEEE Vehicular Technology Conference (VTC) 1998, pp. 552-556, 1998.

[9] Minisry of Healsth, Labor and Welfare (Japan) Guidelines for Safety Management of Medical Information Systems Ver. 4.1 (in Japanese), p. 43, 2010.

Takato Kudou

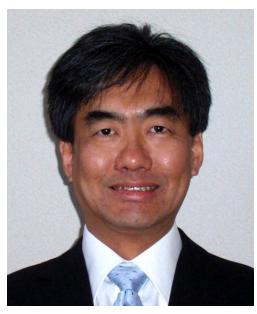

was born in Oita, Japan, in 1963. He received his B. Eng., M. Eng. and D. Eng. degrees in communication engineering, all from Kyushu University, Fukuoka, Japan, in 1985, 1987 and 1990, respectively. From 1990 to 1994 , he was a Research Associate of the Department of Computer Science and Communication Engineering, Kyushu University. In 1994, he joined the Department of Electrical and Electronic Engineering, Oita University, and is currently an Associate Professor of the same University. His research interests have been on electromagnetic direct/inverse scattering and FDTD analysis of the electromagnetic environment. Dr. Kudou is a member of the IEICE of Japan, the IEE of Japan and the Japanese Society for Medical and Biological Engineering. 